\title{
INTRINSIC GEOMETRY OF A NULL HYPERSURFACE *
}

\author{
PAWEe Nurowski $\dagger$ \\ Instytut Fizyki Teoretycznej \\ Uniwersytet Warszawski \\ ul. Hoża 69, Warszawa, Poland \\ and \\ DAVID C. RoBinson $\ddagger$ \\ Department of Mathematics \\ King's College London \\ Strand, London WC2R 2LS, U.K.
}

October 30, 2018

\begin{abstract}
We apply Cartan's method of equivalence to construct invariants of a given null hypersurface in a Lorentzian space-time. This enables us to fully classify the internal geometry of such surfaces and hence solve the local equivalence problem for null hypersurface structures in 4-dimensional Lorentzian space-times.
\end{abstract}

\footnotetext{
*Research supported by Komitet Badań Naukowych (Grant nr 2 P03B 060 17), the Erwin Schrödinger International Institute for Mathematical Physics and the London Mathematical Society.

†e-mail: nurowski@fuw.edu.pl

‡e-mail: david.c.robinson@kcl.ac.uk
} 


\section{Introduction}

The study of the structure of null hypersurfaces in four-dimensional space-time has played a key role in the development of general relativity and the mathematics and physics of gravitation. For example detailed investigations of null hypersurfaces have been neccessary in order to understand the causal structure of space-times, black holes, asymptotically flat systems and gravitational waves. In this paper we shall study and classify the internal geometries of null hypersurfaces.

In J. L. Synge's festshrift volume [7] Roger Penrose distinguished three types of geometries which a null hypersurface $\mathcal{N}$ in 4-dimensional space-time $(\mathcal{M}, g)$, acquires from the ambient Lorentzian geometry. These geometries are associated with the following geometrical structures that are defined on $\mathcal{N}$ :

i) the degenerate metric $g_{\mid \mathcal{N}}$

ii) the concept of an affine parameter along each of the null geodesics from the 2-parameter family ruling $\mathcal{N}$

iii) the concept of parallel transport for tangent vectors to $\mathcal{N}$ along each of the null geodesics.

Penrose's three geometries are then:

$$
\begin{aligned}
& \text { I - the geometry of structure i), } \\
& \text { II - the geometry of structures i) and ii), } \\
& \text { III - the geometry of structures i) and iii). }
\end{aligned}
$$

In this paper we study the weakest of the Penrose geometries, I - geometry. In this context it is convenient to use the following definition of a null hypersurface.

Definition 1 A null hypersurface in an oriented and time oriented space-time $(\mathcal{M}, g)$ is a 3 dimensional submanifold $\mathcal{N}$ in $\mathcal{M}$ which is such that the restricted metric $g_{\mid \mathcal{N}}$ is degenerate.

It follows that if the metric $g$ has signature $(+++-)$ then the signature of $g_{\mid \mathcal{N}}$ is $(++0)$.

Since in this paper we are investigating only Penrose's I - geometry it is convenient to introduce the concept of a null hypersurface structure (NHS).

Definition 2 A null hypersurface structure (NHS) is a 3-dimensional oriented manifold $\mathcal{N}$ equipped with a degenerate metric $h$ of signature $(++0)$.

Note that any null hypersurface in space-time defines an NHS.

Our main aim in this paper is to solve the local equivalence problem for NHS's by using the methods developed by E. Cartan (a helpful modern exposition has been presented by Olver [6]). Consequently, we introduce the following definitions.

Definition 3 Two NHSs $\left(\mathcal{N}_{1}, h_{1}\right)$ and $\left(\mathcal{N}_{2}, h_{2}\right)$ are (locally) equivalent if and only if there exists a (local) diffeomorphism

$$
\psi: \mathcal{N}_{1} \rightarrow \mathcal{N}_{2}
$$

such that

$$
\psi^{*} h_{2}=h_{1} .
$$


Definition 4 A diffeomorphism $\psi$ is called a symmetry of a $N H S(\mathcal{N}, h)$ iff

$$
\psi^{*} h=h .
$$

The local version of this definition is:

Definition 5 A vector field $X$ on $\mathcal{N}$ is an infinitesimal symmetry of a $N H S(\mathcal{N}, h)$ iff

$$
\mathcal{L}_{X} h=0 .
$$

It follows that the set of all infinitesimal symmetries naturally has the structure of a Lie algebra; the Lie algebra of symmetries of the NHS.

Cartan's method for dealing with local equivalence problems is explained fully in Reference [6]. Here we outline, using the notation of that book, only the basic ideas and the definitions which are particularly important in the sequel.

Given a geometrical structure on a manifold, the first step is to redefine it in terms of a coframe given up to certain transformations. More formally, with each geometrical structure one associates an equivalence class of coframes $\left[\left(\omega^{i}\right)\right]=\left[\left(\omega^{1}, \omega^{2}, \ldots, \omega^{n}\right)\right]$, with an equivalence relation such that a coframe $\left(\omega^{i}\right)$ is equivalent to a coframe $\left(\tilde{\omega}^{i}\right)$ if and only if there exists a function $\left(a^{i}{ }_{j}\right)$ with values in a Lie subgroup $G$ of $\mathbf{G L}(n, \mathbf{R})$ such that

$$
\tilde{\omega}^{i}=a^{i}{ }_{j} \omega^{j}
$$

The group $G$ is totally characterized by the geometrical structure considered. The structure is then callled a $G$-structure.

Now, the equivalence problem for two $G$-structures translates into a problem of $G$-equivalence of the corresponding coframes. Such a problem may have two, qualitatively different, outcomes. Either a $G$-structure admits an infinite dimensional group of local symmetries or it does not. In the first case the structure is said to be involutive (or in involution).

Cartan's method provides an algorithm for determining whether a given $G$-structure is in involution or not. The only operations one uses to determine this are differentiation and linear algebra. By means of them, given a $G$-structure on an $n$-dimensional manifold, one calculates the so called degree of indeterminacy of the $G$-structure, $r^{(1)}$, and $n-1$ parameters $s_{i}^{\prime}$, called the Cartan characters of the $G$-structure. The $n$th Cartan character $s_{n}^{\prime}=r-s_{1}^{\prime}-s_{2}^{\prime}-\ldots-s_{n-1}^{\prime}$ is defined in terms of the previous $n-1$ ones and the dimension $r$ of the group $G$. Cartan's result is that the $G$-structure is in involution if the following equality

$$
1 s_{1}^{\prime}+2 s_{2}^{\prime}+3 s_{3}^{\prime}+\ldots+n s_{n}^{\prime}=r^{(1)},
$$

called the Cartan test, holds. Otherwise the $G$-structure is not involutive.

In the involutive case Cartan's theorems give the solution to the equivalence problem. In particular, they state that if $s_{k}^{\prime}$ is the last nonvanishing Cartan character then the set of analytic morphisms transforming a given $G$-structure to itself depends on $s_{k}^{\prime}$ analytic functions of $k$ variables.

If a $G$-structure is not involutive then, by means of the techniques called absorption, normalization and prolongation, one reduces the original $G$-equivalence problem for the initial $G$-structure either to an $\{e\}$-equivalence problem (possibly on a new manifold) or to a new problem for a $G_{1}$-structure with a new group $G_{1}$.

If it happens that the $G$-equivalence problem is reduced to an $\{e\}$-problem then Cartan's theorems provide a simple method for the construction of all the invariants of the original geometrical structure. If one ends with a new $G_{1}$-structure then one asks whether it is involutive or whether it can be 
further reduced to an $\{e\}$-structure. Cartan's theorems state that, under certain regularity assumptions, each $G$-equivalence problem, after a finite number $p$ of steps, will finally reduce either to an $\{e\}$-structure or to a $G_{p}$-structure, which is involutive. In the case of an $\{e\}$-structure Cartan gives, as before, a simple method for constructing all the invariants of the original geometrical structure. In the involutive situation two cases may occur. In the first case, the geometrical structure in question is unique modulo a local $G$-equivalence. In the second case it is not, and Cartan's method produces all the $G$-invariants. In both cases, if we denote the last nonvanishing Cartan character for the final involutive $G_{p}$ structure by $s_{k}^{\prime}$, then the set of analytic morphisms transforming a given $G$-structure to itself depends on $s_{k}^{\prime}$ analytic functions of $k$ variables.

It may be helpful at this point to note that a number of authors have applied Cartan's method of equivalence to the Lorentzian 4-metric of general relativity. Two examples of such work are given by references [2, [4]. We also note that geometrical structures, in Cartan's sense, induced on null hypersurfaces in $n$-dimensional space-times have been investigated in reference [3].

In the next section we outline the basic formalism which we use in this paper. The following section contains a discussion of the G-structure - a null hypersurface structure - to which we shall apply Cartan's method. Section 4 contains the application of the equivalence method. The main results of the paper are stated as a series of propositions in that Section. The final Section contains some brief comments on this work. In addition there are two appendices. Appendix A contains a description of null hypersurfaces structures with zero complex expansion, non-zero shear and three dimensional symmetry groups. Finally the results of the classification computed in Section 4 are summarized diagramatically in Appendix B.

\section{Space-time formalism}

In this section we outline the space-time formalism which we shall use in this paper. In general we follow the conventions and notation of Reference [5] and use the Newman-Penrose formalism as it is presented in that reference. Consider a 4 -dimensional space-time $(\mathcal{M}, g)$ with a metric $g$ of a signature $(+++-)$. Let $\left(e_{1}, e_{2}, e_{3}, e_{4}\right)=(m, \bar{m}, l, k)$ be a null tetrad on $\mathcal{M}$ with dual $(M, \bar{M}, L, K)$ so that $g=2(M \bar{M}-L K)$. For such a space-time the first Cartan structure equations can be written as follows:

$$
\begin{aligned}
\mathrm{d} M & =-\Gamma_{21} \wedge M-\Gamma_{23} \wedge L-\Gamma_{24} \wedge K, \\
\mathrm{~d} L & =\Gamma_{41} \wedge M+\Gamma_{42} \wedge \bar{M}+\Gamma_{43} \wedge L, \\
\mathrm{~d} K & =\Gamma_{31} \wedge M+\Gamma_{32} \wedge \bar{M}+\Gamma_{34} \wedge K,
\end{aligned}
$$

where the connection 1-forms $\Gamma_{i j},(i, j=1,2,3,4)$ satisfy $\Gamma_{i j}=-\Gamma_{j i}$ and a change of indicies $1 \leftrightarrow 2$ implies complex conjugation (e.g. $\Gamma_{13}$ is the complex conjugate of $\Gamma_{23}$ ). In terms of Newman-Penrose coefficients (see Ref. [5] pp. 82-87)

$$
\begin{gathered}
\Gamma_{41}=\sigma M+\rho \bar{M}+\tau L+\kappa K, \\
\Gamma_{23}=\mu M+\lambda \bar{M}+\nu L+\pi K, \\
\frac{1}{2}\left(\Gamma_{12}+\Gamma_{34}\right)=-\beta M-\alpha \bar{M}-\gamma L-\epsilon K .
\end{gathered}
$$

With this notation equations (2.1) read

$$
\begin{aligned}
\mathrm{d} M & =(\alpha-\bar{\beta}) M \wedge \bar{M}+(\gamma-\bar{\gamma}-\mu) M \wedge L-\lambda \bar{M} \wedge L+(\varepsilon-\bar{\varepsilon}+\bar{\rho}) M \wedge K+\bar{\sigma} \bar{M} \wedge K+(\bar{\tau}+\pi) L \wedge K, \\
\mathrm{~d} L & =(\bar{\rho}-\rho) M \wedge \bar{M}+(\bar{\alpha}+\beta-\tau) M \wedge L+(\alpha+\bar{\beta}-\bar{\tau}) \bar{M} \wedge L+\kappa K \wedge M+\bar{\kappa} K \wedge \bar{M}+(\varepsilon+\bar{\varepsilon}) K \wedge L(2.3) \\
\mathrm{d} K & =(\bar{\mu}-\mu) M \wedge \bar{M}+\bar{\nu} M \wedge L+\nu \bar{M} \wedge L+(\bar{\pi}-\beta-\bar{\alpha}) M \wedge K+(\pi-\alpha-\bar{\beta}) \bar{M} \wedge K+(\gamma+\bar{\gamma}) K \wedge L .
\end{aligned}
$$


The second Cartan structure equations are:

$$
\begin{gathered}
\mathrm{d} \Gamma_{23}=\left(\Gamma_{12}+\Gamma_{34}\right) \wedge \Gamma_{23}+\Psi_{4} \bar{M} \wedge L+\Psi_{3}(K \wedge L-M \wedge \bar{M})+\left(\Psi_{2}+\frac{1}{12} R\right) K \wedge M \\
+\frac{1}{2} S_{33} M \wedge L+\frac{1}{2} S_{32}(K \wedge L+M \wedge \bar{M})+\frac{1}{2} S_{22} K \wedge \bar{M} \\
\mathrm{~d} \Gamma_{14}=\Gamma_{14} \wedge\left(\Gamma_{12}+\Gamma_{34}\right)+\left(-\Psi_{2}-\frac{1}{12} R\right) \bar{M} \wedge L-\Psi_{1}(K \wedge L-M \wedge \bar{M})-\Psi_{0} K \wedge M \\
-\frac{1}{2} S_{11} M \wedge L-\frac{1}{2} S_{41}(K \wedge L+M \wedge \bar{M})-\frac{1}{2} S_{44} K \wedge \bar{M} \\
\frac{1}{2}\left(\mathrm{~d} \Gamma_{12}+\mathrm{d} \Gamma_{34}\right)=\Gamma_{23} \wedge \Gamma_{14}-\Psi_{3} \bar{M} \wedge L-\left(\Psi_{2}-\frac{1}{24} R\right)(K \wedge L-M \wedge \bar{M})-\Psi_{1} K \wedge M \\
-\frac{1}{2} S_{31} M \wedge L-\frac{1}{4}\left(S_{12}+S_{34}\right)(K \wedge L+M \wedge \bar{M})-\frac{1}{2} S_{42} K \wedge \bar{M},
\end{gathered}
$$

where we have introduced the (spinorial) Weyl tensor coefficients $\Psi_{\mu},(\mu=0,1,2,3,4)$ and the traceless part of the Ricci tensor $S_{i j}=R_{i j}-g_{i j} R / 4$.

It should also be noted here that the structure equations (2.1), (2.4) imply the Newman-Penrose equations (7.28)-(7.71) of Ref. [5].

Since we shall be considering the geometry of null hypersurfaces ruled by null geodesics with null tangent vector $k$ we shall also need to employ the null rotations which preserve the direction of $k$.

Let $A>0, \phi$ (real) and $z$ (complex) be functions on $\mathcal{M}$. Then the action of the Lorentz transformations on the null coframe, which preserve the $k$ direction is given by

$$
\begin{gathered}
M \rightarrow \mathrm{e}^{i \phi}[M+z L], \\
L \rightarrow A^{-1} L, \\
K \rightarrow A[K+z \bar{z} L+z \bar{M}+\bar{z} M] .
\end{gathered}
$$

The corresponding transformations of the connection 1-forms are given by:

$$
\begin{gathered}
\Gamma_{41} \rightarrow A^{-1} \mathrm{e}^{-i \phi} \Gamma_{41} \\
\Gamma_{23} \rightarrow A \mathrm{e}^{i \phi}\left[\Gamma_{23}+z\left(\Gamma_{12}+\Gamma_{34}\right)-z^{2} \Gamma_{14}-\mathrm{d} z\right] \\
\Gamma_{12}+\Gamma_{34} \rightarrow \Gamma_{12}+\Gamma_{34}+2 z \Gamma_{41}+\mathrm{d} \log A+i \mathrm{~d} \phi .
\end{gathered}
$$

In subsequent sections we shall use these null frames and equations restricted to the null hypersurface $\mathcal{N}$. We shall not distinguish notationally between space-time and null hypersurface quantities but the distinction will be clear from the context.

\section{G-structure for null hypersurface structures (NHS's)}

To apply Cartan's method to a NHS $(\mathcal{N}, h)$ we first need to reformulate the definition of a NHS in terms of a co-frame defined, up to appropriate transformations, on $\mathcal{N}$. To achieve this, recall that each NHS $(\mathcal{N}, h)$ has a metric tensor $h$ of signature $(++0)$. Thus, locally, it can be written in the form $h=2 M \bar{M}$, where $M$ is a complex-valued 1-form on $\mathcal{N}$, such that $M \wedge \bar{M} \neq 0$. On $\mathcal{N}$ the 1-form $M$ and its complex conjugate $\bar{M}$ may always be augmented by a real-valued 1-form $K$ such that $M \wedge \bar{M} \wedge K \neq 0$. Since $\mathcal{N}$ is oriented we demand that $(\operatorname{Re} M, \operatorname{Im} M, K)$ form a coframe on $\mathcal{N}$ which agrees with the orientation. This coframe is given up to the following transformations (null rotations on $\mathcal{N})$ :

$$
M \rightarrow \mathrm{e}^{i \phi} M, \quad K \rightarrow A[K+z \bar{M}+\bar{z} M]
$$


where $A>0, \phi$ (real) and $z$ (complex) are functions on $\mathcal{N}$. The $G$-structure we shall consider corresponds to this group, $G$, of null rotations. This leads to the following reformulation of the definition for NHSs:

Definition 6 A null hypersurface structure is a 3-dimensional manifold $\mathcal{N}$ equipped with an equivalence class of 1-forms $[(M, K)]$ such that

$M$ is complex- and $K$ is real-valued,

$M \wedge \bar{M} \wedge K \neq 0$ at every point of $\mathcal{N}$,

two pairs $(M, K)$ and $\left(M_{1}, K_{1}\right)$ are equivalent if and only if they are related by transformations (3.1).

Definition 7 Two null hypersurface structures $(\mathcal{N},[(M, K)])$ and $\left(\mathcal{N}_{1},\left[\left(M_{1}, K_{1}\right)\right]\right)$ are (locally) equivalent iff there exists a (local) diffeomorphism $\varphi: \mathcal{N} \rightarrow \mathcal{N}_{1}$ and a function $\phi: \mathcal{N} \rightarrow[0,2 \pi[$ such that $\varphi^{*} M_{1}=\mathrm{e}^{i \phi} M$.

If there exists a (local) diffeomorphism $\varphi: \mathcal{N} \rightarrow \mathcal{N}$ such that $\varphi^{*} M=\mathrm{e}^{i \phi} M$ where $\phi$ is a real function, then such $a \varphi$ is called a (local) symmetry of a null hypersurface structure $(\mathcal{N},[(M, K)])$. An infinitesimal symmetry of $(\mathcal{N},[(M, K)])$ is a real vector field $X$ on $\mathcal{N}$ such that $\mathcal{L}_{X} M=i t M$, where $t$ is a real function on $\mathcal{N}$.

Let $(\mathcal{N},[(M, K)])$ be a null hypersurface structure as defined above. Let $(M, K)$ be a particular representative of the class $[(M, K)])$. Given a pair $(M, K)$ we have its differentials which, when decomposed onto the basis of 2-forms spanned by $M \wedge \bar{M}, M \wedge K$ and $\bar{M} \wedge K$, can be written as follows:

$$
\begin{gathered}
\mathrm{d} M=(\alpha-\bar{\beta}) M \wedge \bar{M}+(\varepsilon-\bar{\varepsilon}+\rho) M \wedge K+\bar{\sigma} \bar{M} \wedge K, \\
\mathrm{~d} K=(\bar{\mu}-\mu) M \wedge \bar{M}+(\bar{\pi}-\beta-\bar{\alpha}) M \wedge K+(\pi-\bar{\beta}-\alpha) \bar{M} \wedge K .
\end{gathered}
$$

Here $\alpha, \beta, \varepsilon, \mu, \pi$ are complex functions and $\rho$ is a real function on $\mathcal{N}$.

To describe the above differentials one needs fewer functions than we have introduced but we write the equations this way so that they agree notationally with equations (2.3) of Section 2.

We recall that any null hypersurface $\mathcal{N}$ in a space-time $(\mathcal{M}, g)$ has its natural null hypersurface structure $\left(\mathcal{N}, h=g_{\mid \mathcal{N}}\right)$. If one has a NHS which originates from a null hypersurface $\mathcal{N}$ in $(\mathcal{M}, g)$ then one can locally introduce a null coframe $(M, \bar{M}, L, K)$ on $\mathcal{M}$ such that the metric $g$ can be written as

$$
g=2(M \bar{M}-L K)
$$

and the null hypersurface $\mathcal{N}$ can be locally defined as a 3 -dimensional surface in $\mathcal{M}$ such that $L_{\mid \mathcal{N}} \equiv 0$. Such a null tetrad satisfies the equations of Section 2. Now, if one restricts these equations to the null hypersurface $\mathcal{N}$ on which $L \equiv 0$, then the second of these equations imply that $\rho-\bar{\rho} \equiv \kappa \equiv 0$ on $\mathcal{N}$. The first and the third of these equations coincide with equations (3.2)-(3.3). Thus, although the notation in equations (3.2)-(3.3) is redundant, it has the advantage that the functions appearing in them can be interpreted as the standard Newman-Penrose coefficients restricted to a null hypersurface in the case where a NHS originates from a null hypersurface in a space-time.

Hence we use the notation of equations (2.2) to write, on $\mathcal{N}$,

$$
\begin{gathered}
\Gamma_{41}=\sigma M+\rho \bar{M}, \\
\Gamma_{23}=\mu M+\lambda \bar{M}+\pi K, \\
\frac{1}{2}\left(\Gamma_{12}+\Gamma_{34}\right)=-\beta M-\alpha \bar{M}-\epsilon K .
\end{gathered}
$$


The functional coefficients in equations (3.2)-(3.3) depend on the choice of a representative $(M, K)$ from the class $[(M, K)]$. In particular we have:

Proposition 1 Under the gauge transformations (3.1) the coefficients $\rho$ and $\sigma$ in equations (3.8)3.5) transform according to

$$
\sigma \rightarrow A^{-1} \mathrm{e}^{-2 i \phi} \sigma, \quad \rho \rightarrow A^{-1} \rho .
$$

It follows from this proposition that although $\rho$ and $\sigma$ are not well defined objects for a given NHS, their vanishing or not is an invariant property characterizing a NHS. Thus, it is clear that NHSs split into four disjoint classes which can not be transformed into each other by diffeomorphisms. These classes are characterized by

1) $\sigma=\rho=0$ on $\mathcal{N}$

2) $\rho=0$ and $\sigma \neq 0$ on $\mathcal{N}$

3) $\sigma \rho \neq 0$ on $\mathcal{N}$

4) $\sigma=0$ and $\rho \neq 0$ on $\mathcal{N}$

We shall now apply Cartan's method to study the local equivalence problem, considering each of these cases in turn. The order in which we consider these inequivalent classes is determined by the way Cartan's method applies to each of them.

\section{Cartan's invariants for the I - geometry of a null hyper- surface}

In this section we apply Cartan's method and solve the local equivalence problem for null hypersurface structures $(\mathcal{N},[(M, K)])$ as defined in the previous section. A particular representative of $[(M, K)]$, say $(M, K)$, satisfies equations (3.2)-(3.3) and the equations implied by them.

To construct the Cartan invariants of a NHS we shall need to consider $G$-bundles or sub-bundles of $G$-bundles over $\mathcal{N}$. The highest dimensional such bundle will be $\mathcal{N} \times \mathcal{F}$, where we shall denote the fibre coordinates of $\mathcal{F}$ by the real positive $A$, the complex $z$ and the real $\phi \in[0,2 \pi[$. On occasion subbundles of $\mathcal{N} \times \mathcal{F}$ with a subset of fibre coordinates will be used. Since prolongations involve replacing gauge group functions by coordinates on $G$-bundles over $\mathcal{N}$ we shall follow the standard shorthand of using the same symbols $A, z$ and $\phi$ for functions on $\mathcal{N}$ and coordinates on the bundles over $\mathcal{N}$. The meaning of the symbol will be clear from the context. On $\mathcal{N} \times \mathcal{F}$ we will have the lifted coframe", denoted with primes,

$$
M^{\prime}=\mathrm{e}^{i \phi} M, \quad K^{\prime}=A[K+z \bar{M}+\bar{z} M]
$$

(and analogously on sub-bundles). Now the usual Cartan procedure of absorbtion and normalization ([6] pp. 307-309) is applied to get the Cartan invariants of the hypersurface. First, we note that the differential of $M^{\prime}$ can be written in the form

$$
\mathrm{d} M^{\prime}=i \omega^{1} \wedge M^{\prime}+\frac{\mathrm{e}^{2 i \phi}}{A} \bar{\sigma} \bar{M}^{\prime} \wedge K^{\prime}+\frac{\rho}{A} M^{\prime} \wedge K^{\prime} .
$$

\footnotetext{
${ }^{1}$ We use the terminology of $[6]$.
} 
The 1 -form $\omega^{1}$ is not unique. It is fixed by requiring that both $\omega^{1}$ and the coefficient at the $M^{\prime} \wedge K^{\prime}$ term are real. Then, $\omega^{1}$ reads:

$$
\omega^{1}=\mathrm{d} \phi+i \Gamma_{21}+i(\bar{z} \bar{\sigma}-z \rho) \bar{M}+i(\bar{z} \bar{\rho}-z \sigma) M .
$$

Equation (4.2) implies that the Cartan procedure for getting the invariants branches according as $\sigma \rho$ is zero or not.

\subsection{Class 1: $\sigma=\rho=0$ on $\mathcal{N}$}

This is a physically interesting case including as it does the wave-fronts of plane fronted waves, null infinity in the zero-divergence conformal gauge and horizons, including the recently introduced 'isolated horizons' [1].

In this case equation (4.2) becomes

$$
\mathrm{d} M^{\prime}=i \omega^{1} \wedge M^{\prime}
$$

with

$$
\omega^{1}=\mathrm{d} \phi+i \Gamma_{21} .
$$

Calculation of the differential of $K^{\prime}$ in this case yields

$$
\mathrm{d} K^{\prime}=\omega^{2} \wedge K^{\prime}+\bar{\omega}^{3} \wedge M^{\prime}+\omega^{3} \wedge \bar{M}^{\prime}
$$

where

$$
\begin{gathered}
\omega^{2}=\mathrm{d} \log A+\Gamma_{34}+b K+c M+\bar{c} \bar{M} \\
\omega^{3}=A \mathrm{e}^{i \phi}\left[\mathrm{d} z+\Gamma_{32}-z\left(\Gamma_{12}+\Gamma_{34}\right)+\left(h+\frac{\bar{c} \bar{z}-c z}{2}\right) M+f \bar{M}+(\bar{c}-b z) K\right],
\end{gathered}
$$

where we have introduced unknown functions $b, h$ (real) and $f, c$ (complex).

Calculations using equations (4.4), (4.6) show that the original system $(\mathcal{N},[(M, K)])$ does not pass the Cartan test (cf. 6, pp. 350-355). Hence it must be prolonged.

The simplest method of prolongation is to first consider a new manifold $\mathcal{N} \times \mathbf{S}^{1}$ parametrized by $(p, \phi)$, where $p \in \mathcal{N}$ and $\phi \in\left[0,2 \pi\left[\right.\right.$. On this manifold one considers the system of 1 -forms $\left(M^{\prime}, K, \omega^{1}\right)$ such that

i) $M^{\prime}$ is complex-, $K$ and $\omega^{1}$ are real-valued

ii) $M^{\prime} \wedge \bar{M}^{\prime} \wedge K \wedge \omega^{1} \neq 0$ on $\mathcal{N} \times \mathbf{S}^{1}$

iii) $M^{\prime}=\mathrm{e}^{i \phi} M, \omega^{1}=\mathrm{d} \phi+i \Gamma_{21}$, where the forms $M, K$ and $\Gamma_{i j}=-\Gamma_{j i}$ satisfy (3.4) with $\Gamma_{41}=0$ on $\mathcal{N}$ and are the lifts to $\mathcal{N} \times \mathbf{S}^{1}$ of the corresponding forms on $\mathcal{N}$.

iv) forms $\left(M^{\prime}, K, \omega^{1}\right)$ are given up to the following transformations

$$
M^{\prime} \rightarrow M^{\prime}, \quad K \rightarrow A\left[K+z \mathrm{e}^{i \phi} \bar{M}^{\prime}+\bar{z} \mathrm{e}^{-i \phi} M^{\prime}\right], \quad \omega^{1} \rightarrow \omega^{1},
$$

where $A>0$ and $z$ are real- and complex functions on $\mathcal{N} \times \mathbf{S}^{1}$.

Now we have a new equivalence problem on $\mathcal{N} \times \mathbf{S}^{1}$ with group $G_{1}$ given by the transformations in iv). We now prolong again. Consider now the manifold $\tilde{\mathcal{N}}=\mathcal{N} \times \mathbf{S}^{1} \times \mathbf{R}_{+} \times \mathbf{C}$ parametrized 
by $(q, A, z)$, where $q \in \mathcal{N} \times \mathbf{S}^{1}$. The $G_{1}$-structure, when extended to $\tilde{\mathcal{N}}$, has the lifted coframe $\left(M^{\prime}, \bar{M}^{\prime}, K^{\prime}, \omega^{1}\right)$ with the following differentials:

$$
\begin{gathered}
\mathrm{d} M^{\prime}=i \omega^{1} \wedge M^{\prime}, \\
\mathrm{d} K^{\prime}=\omega^{2} \wedge K^{\prime}+\bar{\omega}^{3} \wedge M^{\prime}+\omega^{3} \wedge \bar{M}^{\prime}, \\
\mathrm{d} \omega^{1}=i\left[-\bar{\Psi}_{2}-\Psi_{2}+\frac{S_{12}+S_{34}}{2}+\frac{R}{12}\right] M^{\prime} \wedge \bar{M}^{\prime},
\end{gathered}
$$

where

$$
\begin{gathered}
\omega^{2}=\mathrm{d} \log A+\Gamma_{34}+b K+c M+\bar{c} \bar{M} \\
\omega^{3}=A \mathrm{e}^{i \phi}\left[\mathrm{d} z+\Gamma_{32}-z\left(\Gamma_{12}+\Gamma_{34}\right)+\left(h+\frac{\bar{c} \bar{z}-c z}{2}\right) M+f \bar{M}+(\bar{c}-b z) K\right] .
\end{gathered}
$$

In these formulae $b, h$ (real) and $c, f$ (complex) are undetermined functions on $\mathcal{N} \times \mathbf{S}^{1}, \Psi_{\mu}, S_{i j}$ and $R$ are the Weyl tensor coefficients, the traceless Ricci tensor and the Ricci scalar restricted to the null hypersurface and lifted to $\mathcal{N} \times \mathbf{S}^{1}$ (by the usual demand that they be constant along the $\phi$ direction).

The system (4.9) has no essential torsions. It passes the Cartan test with the following values of the Cartan parameters:

i) dimension of $\mathcal{N} \times \mathbf{S}^{1}: \mathrm{n}=4$,

ii) dimension of the structure group: $r=3$

iii) degree of indeterminacy: $r^{(1)}=6$,

iv) Cartan characters: $s_{1}^{\prime}=1, s_{2}^{\prime}=1, s_{3}^{\prime}=1, s_{4}^{\prime}=0$.

Now, assuming that our lifted coframe $\left(M^{\prime}, \bar{M}^{\prime}, K^{\prime}, \omega^{1}\right)$ is regular and analytic we can apply Cartan's theorem (Theorem 11.16 of Ref. [6]) to decide when two given NHS's with vanishing relative invariants $\sigma$ and $\rho$ are locally equivalent.

First we observe, from equations (4.9), that the only invariant of a hypersurface with $\sigma=\rho=0$ is

$$
I=-\bar{\Psi}_{2}-\Psi_{2}+\frac{S_{12}+S_{34}}{2}+\frac{R}{12}
$$

and the invariants derived from it by differentiation. A simple geometric interpretation of $I$ is as follows. Consider a basis $\left(m^{\prime}, \bar{m}^{\prime}, k^{\prime}, o\right)$ dual to $\left(M^{\prime}, \bar{M}^{\prime}, K^{\prime}, \omega^{1}\right)$ on $\mathcal{N} \times \mathbf{S}^{1}$. The first equation in the system (4.9) guarantees that $\mathcal{N} \times \mathbf{S}^{1}$ is foliated by two-dimensional fibres tangent to vector fields $k^{\prime}$ and $o$. Moreover, a $(++00)$-signature metric $\hat{h}=2 M^{\prime} \bar{M}^{\prime}$ on $\mathcal{N} \times \mathbf{S}^{1}$ has vanishing Lie derivative along the directions of $k^{\prime}$ and $o$. Thus, the metric $\hat{h}$ projects down to a well defined metric $h$ on a 2-dimensional manifold $\mathcal{N} \times \mathbf{S}^{1} / \sim$ of the leaves of the foliation?. It can be easily checked that $I$ is the Gaussian curvature of $h$.

The theorem of Cartan (Thm. 11.16 of Ref. [6]) implies that, in the fully regular, analytic case there always exist a choice of $A$ and $z$ such that $\mathrm{d} K^{\prime}=0$ in equations (4.9). Such a choice of $K^{\prime}$

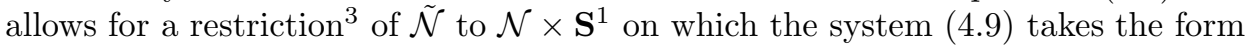

$$
\begin{gathered}
\mathrm{d} M^{\prime}=i \omega^{1} \wedge M^{\prime}, \\
\mathrm{d} K^{\prime}=0, \\
\mathrm{~d} \omega^{1}=i I M^{\prime} \wedge \bar{M}^{\prime} .
\end{gathered}
$$

\footnotetext{
${ }^{2}$ In other words, any 2-dimensional surface $\mathcal{S}$ transversal to the leaves of the foliation acquires a Riemannian metric $\hat{h}_{\mid \mathcal{S}}$ by restricting $\hat{h}$ to $\mathcal{S}$. The vanishing of the Lie derivatives means that for any $\mathcal{S}$ the metrics $\hat{h}_{\mid \mathcal{S}}$ are locally isometric.

${ }^{3}$ By a 'restriction' here we mean such a choice of a section of the bundle $\mathcal{N} \times \mathbf{S}^{1} \rightarrow \tilde{\mathcal{N}}$ on which d $K^{\prime}=0$ holds. This section is then identified with $\mathcal{N} \times \mathbf{S}^{1}$.
} 
It is easy to integrate these equations. The result is:

$$
\begin{gathered}
M^{\prime}=\frac{\mathrm{e}^{i \phi}}{P} \mathrm{~d} \xi, \\
K^{\prime}=\mathrm{d} r, \\
\omega^{1}=\mathrm{d} \phi+i\left[(\log P)_{\bar{\xi}} \mathrm{d} \bar{\xi}-(\log P)_{\xi} \mathrm{d} \xi\right], \\
I=\triangle \log P, \quad \triangle=2 P^{2} \partial_{\xi} \partial_{\bar{\xi}},
\end{gathered}
$$

where $(\xi, \bar{\xi}, r, \phi)$ constitutes a coordinate system on $\mathcal{N} \times \mathbf{S}^{1}, P=P(\xi, \bar{\xi})$ is a real function, and subscripts such as $\xi$ denote partial derivatives. Thus we have the following proposition.

Proposition 2 Any analytic $N H S(\mathcal{N},[(M, K)])$ with vanishing relative invariants $\sigma$ and $\rho$ is locally equivalent to the one defined by forms

$$
M=\frac{1}{P} \mathrm{~d} \xi, \quad K=\mathrm{d} r,
$$

where $P=P(\xi, \bar{\xi})$ is a real function. The lowest order Cartan invariant for such NHS's is

$$
I=\triangle \log P, \quad \triangle=2 P^{2} \partial_{\xi} \partial_{\bar{\xi}} .
$$

$I=2 c=$ const if and only if the function $P=1+c \xi \bar{\xi}$. If $I \neq$ const, then the higher order invariants are

i) first order: $I_{1}=P I_{\xi} \mathrm{e}^{-i \phi}$

ii) second order: $I_{2}=P\left(P I_{\xi \xi}+2 P_{\xi} I_{\xi}\right) \mathrm{e}^{-2 i \phi}$ and $I_{3}=\frac{1}{2} \triangle I=\frac{1}{2} \triangle \triangle \log P$,

where the $\phi$ variable is related to the fourth dimension of the prolonged manifold $\mathcal{N} \times \mathbf{S}^{1}$.

The local group of symmetries for each such structure is infinite dimensional; the set of analytic self-equivalences depends on one real function of three real variables.

\subsection{Class 2: $\sigma=0, \rho \neq 0$ on $\mathcal{N}$}

In this case equations (4.2)-(4.3) become, on $\mathcal{N} \times \mathcal{F}$,

$$
\mathrm{d} M^{\prime}=i \omega^{1} \wedge M^{\prime}+\frac{\rho}{A} M^{\prime} \wedge K^{\prime},
$$

with

$$
\omega^{1}=\mathrm{d} \phi+i \Gamma_{21}-i z \rho \bar{M}+i \bar{z} \bar{\rho} M .
$$

Thus, we can always normalize the coefficient preceeding $M^{\prime} \wedge K^{\prime}$ to $s= \pm 1=\operatorname{sign}(\rho)$ by choosing a section of $\mathcal{N} \times \mathbf{S}^{1} \times \mathbf{C} \rightarrow \mathcal{N} \times \mathcal{F}$ so that

$$
A=|\rho| .
$$

Using this condition we obtain on $\mathcal{N} \times \mathbf{S}^{1} \times \mathbf{C}$

$$
\begin{gathered}
\mathrm{d} M^{\prime}=i \omega^{1} \wedge M^{\prime}+s M^{\prime} \wedge K^{\prime}, \\
\mathrm{d} K^{\prime}=\bar{\omega}^{2} \wedge M^{\prime}+\omega^{2} \wedge \bar{M}^{\prime},
\end{gathered}
$$

where $\omega^{1}$ is given by 4.14 ,

$\omega^{2}=s \mathrm{e}^{i \phi}\left[\rho \mathrm{d} z+\left(z\left(\Phi_{01}-\Psi_{1}\right)+\rho(2 z \beta-\mu)\right) M+\left(z \Phi_{00}+\bar{\Psi}_{1}-\bar{\Phi}_{01}+\rho(2 z \varepsilon-\pi)\right) K+a M+b \bar{M}\right]$ 
and $a$ is any real function and $b$ is any complex function on $\mathcal{N} \times \mathbf{S}^{1} \times \mathbf{C}$. It turns out that the system (4.16)-(4.17) does not pass Cartan's test. Therefore following the standard procedure we consider the new equivalence problem for the sub-group preserving the normalisation above. Hence we consider a new prolongation from $\mathcal{N}$ to $\mathcal{N} \times \mathbf{S}^{1}$ on which we have

$$
\begin{gathered}
\mathrm{d} M^{\prime}=i \omega^{1} \wedge M^{\prime}+s M^{\prime} \wedge K^{\prime}, \\
\mathrm{d} K^{\prime}=\bar{\omega}^{2} \wedge M^{\prime}+\omega^{2} \wedge \bar{M}^{\prime}, \\
\mathrm{d} \omega^{1}=s i \bar{\omega}^{2} \wedge M^{\prime}-s i \omega^{2} \wedge \bar{M}^{\prime}+i\left(-\bar{\Psi}_{2}-\Psi_{2}+\frac{S_{12}+S_{34}}{2}+\frac{R}{12}+2 a\right) M^{\prime} \wedge \bar{M}^{\prime} .
\end{gathered}
$$

The last of the above equations can be reduced to the form

$$
\mathrm{d} \omega^{1}=s i \bar{\omega}^{2} \wedge M^{\prime}-s i \omega^{2} \wedge \bar{M}^{\prime}
$$

by choosing $a=\frac{1}{2}\left(\bar{\Psi}_{2}+\Psi_{2}-\frac{S_{12}+S_{34}}{2}-\frac{R}{12}\right)$. Now, it can be easily checked that the system given by equations (4.19)-(4.21) on $\mathcal{N} \times \mathbf{S}^{1}$ is in involution (The Cartan parameters are: $n=4, r=2$, $r^{(1)}=2, s_{1}^{\prime}=2, s_{2}^{\prime}=0, s_{3}^{\prime}=0, s_{4}^{\prime}=0$ ), so in the analytic fully regular case, it is gauge equivalent to the system

$$
\begin{gathered}
\mathrm{d} M^{\prime}=i \omega^{1} \wedge M^{\prime}+s M^{\prime} \wedge K^{\prime} \\
\mathrm{d} K^{\prime}=0 \\
\mathrm{~d} \omega^{1}=0 .
\end{gathered}
$$

This means that in this case where $\sigma=0, \rho \neq 0$ we have only two locally non-equivalent null hypersurfaces. They correspond to the choices $s=+1$ and $s=-1$ in equations (4.22).

The system of equations 4.22 can be easily solved. The result is:

$$
\begin{gathered}
M^{\prime}=\mathrm{e}^{i \phi \mp r} \mathrm{~d} \xi \\
K^{\prime}=\mathrm{d} r \\
\omega^{1}=\mathrm{d} \phi,
\end{gathered}
$$

where $(\xi, \bar{\xi}, r, \phi)$ constitutes a coordinate system on $\mathcal{N} \times \mathbf{S}^{1}$. In this way we obtained the following proposition.

Proposition 3 Any analytic null hypersurface structure $(\mathcal{N},[(M, K)])$ with vanishing relative invariant $\sigma$ and nonvanishing $\rho$ is locally equivalent to one of the two NHS's defined by the forms

$$
\begin{gathered}
M=\mathrm{e}^{-r} \mathrm{~d} \xi \\
K=\mathrm{d} r
\end{gathered}
$$

or by the forms

$$
\begin{gathered}
M=\mathrm{e}^{r} \mathrm{~d} \xi \\
K=\mathrm{d} r
\end{gathered}
$$

on a manifold $\mathcal{N}$ with coordinates $(\xi, \bar{\xi}, r)$.

The local group of symmetries for such structures is infinite dimensional; the set of analytic selfequivalences depends on two real functions of one real variable.

We note that past and the future null cones in Minkowski space-time possess the NHS's of Proposition 3 .

In dealing with the last two classes we continue to use methods and notation similar to those used above. However, as the essential features of Cartan's method are algorithmic and repetitive we increasingly abbreviate. The reader interested only in the final results of the calculations will find them stated as propositions at the ends of the sections. 


\subsection{Class 3: $\sigma \neq 0, \rho \neq 0$}

In this, the generic case, we can always normalize the factor of the $\bar{M}^{\prime} \wedge K^{\prime}$ term of (4.2) to 1 . We achieve this by imposing condition $A \mathrm{e}^{2 i \phi}=\sigma$ on the group parameters $A, \phi$. One sees that this condition fixes both $A$ and $\phi$. From now, on we assume that a representative $(M, K)$ defining a null surface structure on $\mathcal{N}$ has been gauged to the form in which equation (3.2) is

$$
\mathrm{d} M=(\alpha-\bar{\beta}) M \wedge \bar{M}+(\varepsilon-\bar{\varepsilon}+\rho) \bar{M} \wedge K+\bar{M} \wedge K .
$$

This condition is equivalent to the statement that $(M, K)$ has been gauged to the form in which

$$
\sigma=1 .
$$

After this has been done, the remaining gauge freedom is

$$
M \rightarrow s M, \quad K \rightarrow K+z \bar{M}+\bar{z} M,
$$

where $s= \pm 1$. Thus we have a new equivalence problem defined by the above transformations. To construct the Cartan invariants we consider a manifold $\mathcal{N} \times \mathbf{C}$ parametrized by $(p, z), p \in \mathcal{N}, z \in \mathbf{C}$. Then a straightforward calculation, using as before lifts of $M$ and $K$, shows that the differential of $M^{\prime}$ is

$$
\mathrm{d} M^{\prime}=s[\bar{z}-z(\rho+\varepsilon-\bar{\varepsilon})+\alpha-\bar{\beta}] M^{\prime} \wedge \bar{M}^{\prime}+(\rho+\varepsilon-\bar{\varepsilon}) M^{\prime} \wedge K^{\prime}+\bar{M}^{\prime} \wedge K^{\prime} .
$$

This equation implies that the function

$$
I_{1}^{0}=\rho+\varepsilon-\bar{\varepsilon}
$$

constitutes an invariant of the NHS

Now, two cases may occur. Either

$$
\text { (i) } \quad\left|I_{1}^{0}\right| \neq 1 \quad \text { (the generic case) }
$$

or

$$
\text { (ii) } \quad\left|I_{1}^{0}\right|=1 \quad \text { (the special case). }
$$

Case (i): $\left|I_{1}^{0}\right| \neq 1$.

If $\left|I_{1}^{0}\right| \neq 1$ we choose

$$
z=\frac{\bar{I}_{1}^{0}(\alpha-\bar{\beta})+\bar{\alpha}-\beta}{\left|I_{1}^{0}\right|^{2}-1}
$$

to reduce equation (4.32) to the form $($ on $\mathcal{N})$

$$
\mathrm{d} M^{\prime}=I_{1}^{0} M^{\prime} \wedge K^{\prime}+\bar{M}^{\prime} \wedge K^{\prime} .
$$

Now, the only remaining freedom is in the choice of a sign $s$. To fix this, we remark that equation (4.35) implies that a gauge can be chosen in which the initial differential forms $(M, K)$ satisfy equations (3.2), (3.3) with $\sigma=1$ and $\alpha=\bar{\beta}$. In such a gauge the differential of the form $K^{\prime}$ is (on $\mathcal{N})$

$$
\mathrm{d} K^{\prime}=i I_{2}^{0} M^{\prime} \wedge \bar{M}^{\prime}+I_{3}^{0} M^{\prime} \wedge K^{\prime}+\bar{I}_{3}^{0} \bar{M}^{\prime} \wedge K^{\prime}
$$

\footnotetext{
${ }^{4}$ Remember that this expression for the invariant $I_{1}^{0}$ is valid only in the gauge $(4.31$.
} 
where the coefficients $I_{i}^{0}$ are:

$$
I_{1}^{0}=\rho+\varepsilon-\bar{\varepsilon}, \quad I_{2}^{0}=i(\mu-\bar{\mu}), \quad I_{3}^{0}=s(\bar{\pi}-2 \bar{\alpha}) .
$$

(Note, that since $\rho \neq 0, I_{1}^{0}+\bar{I}_{1}^{0} \neq 0$ ).

Now, assuming that $I_{3}^{0} \neq 0$ we can fix $s$ in such a way that $s \operatorname{Re}\left[I_{3}^{0}\right]>0\left(\right.$ if $\left.\operatorname{Re}\left[I_{3}^{0}\right] \neq 0\right)$ or $s \operatorname{Im}\left[I_{3}^{0}\right]>0$ (if $\operatorname{Re}\left[I_{3}^{0}\right]=0$ ). If $I_{3}^{0}=0$ there is no way to fix the sign $s$.

We summarize the result of this section in the following proposition.

Proposition 4 The generic NHS $(\mathcal{N},[(M, K)])\left(\sigma \neq 0, \rho \neq 0,\left|I_{1}^{0}\right| \neq 1, I_{1}^{0}+\bar{I}_{1}^{0} \neq 0\right)$ defines invariant forms $(M, K)$ which satisfy

$$
\begin{gathered}
\mathrm{d} M=I_{1}^{0} M \wedge K+\bar{M} \wedge K \\
\mathrm{~d} K=i I_{2}^{0} M \wedge \bar{M}+I_{3}^{0} M \wedge K+\bar{I}_{3}^{0} \bar{M} \wedge K,
\end{gathered}
$$

in a unique way if $I_{3}^{0} \neq 0$, and with a sign ambiguity in the choice of $M$ if $I_{3}^{0}=0$. The scalar invariants of zeroth order for such a NHS are $I_{1}^{0}$ (such that $\left|I_{1}^{0}\right| \neq 1$ ), $I_{2}^{0}$ (real) and $I_{3}^{0}$ (in general complex). All the other invariants are derived from those by means of differentiation.

Any such null hypersurface has a local group of symmetries of dimension no greater than 3.

The system (4.38)-(4.39) is too general to be as easily integrated as was the case in the systems studied in preceding sections. Here we only consider the system (4.38)-(4.39) when it has a 3dimensional group of symmetries acting transitively on $\mathcal{N}$, that is, all the invariants $I_{1}^{0}, I_{2}^{0}$ and $I_{3}^{0}$ are constants on $\mathcal{N}$ - zeroth order NHS's. Then the equations $\mathrm{d}^{2} M=0, \mathrm{~d}^{2} K=0$, consequences of equations (4.38)-(4.39), imply that $I_{2}^{0}=I_{3}^{0}=0$. The other possibility $I_{1}^{0}+\bar{I}_{1}^{0}=I_{3}^{0}=0$ is excluded by the assumption $\rho \neq 0$.

Proposition 5 Any $N H S(\mathcal{N},[(M, K)])$ with $I_{2}^{0}=I_{3}^{0}=0$ and $I_{1}^{0}=a+i b, a, b=$ const $\in \mathbf{R}$ is equivalent to the one defined by forms on $\mathcal{N}$ given by

$$
M=\left[\bar{\xi}+I_{1}^{0} \xi\right] \mathrm{d} r+\mathrm{d} \xi, \quad K=\mathrm{d} r .
$$

The three dimensional algebra of infinitesimal symmetries of such a NHS is of Bianchi type

i) $I V$ iff $b= \pm 1$,

ii) $V I_{-h}, h=\frac{a^{2}}{b^{2}-1}$ iff $|b|<1$,

iii) $V I I_{h}, h=\frac{a^{2}}{b^{2}-1}$ iff $|b|>1$.

Thus, there are only two nonequivalent generic NHS's of zeroth order admitting the symmetry algebra of Bianchi type $I V$. For each value of the parameter $h$, there is a one-parameter family of nonequivalent NHS's of zeroth order of Bianchi types $V I_{-h}$ and $V I I_{h}$.

Case (ii): $\left|I_{1}^{0}\right|=1$.

If $\left|I_{1}^{0}\right|=1$ there exists a real function $t: \mathcal{N} \rightarrow\left[0,2 \pi\left[\right.\right.$ such that $I_{1}^{0}=\mathrm{e}^{i t}$. With this choice equation (4.32) assumes the form

$$
\mathrm{d} M^{\prime}=s\left[\bar{z}-z \mathrm{e}^{i t}+\alpha-\bar{\beta}\right] M^{\prime} \wedge \bar{M}^{\prime}+\mathrm{e}^{i t} M^{\prime} \wedge K^{\prime}+\bar{M}^{\prime} \wedge K^{\prime} .
$$

Now the following two cases may occur

ii.a) $t \neq \pi$

ii.b) $t=\pi$.

\footnotetext{
${ }^{5}$ Hence the classification of inequivalent generic zeroth order NHS's is in correspondence with the Bianchi classification of 3-dimensional Lie algebras
} 
Case ii.a) $(t \neq \pi)$

Let $\operatorname{Re}[\alpha-\bar{\beta}]=A, \operatorname{Im}[\alpha-\bar{\beta}]=B, \operatorname{Re}[z]=\zeta, \operatorname{Im}[z]=\eta$. Since $t \neq \pi$ we can choose the function $\eta$ in the form

$$
\eta=\frac{B-\zeta \sin t}{1+\cos t}
$$

Such a choice of $\eta$ corresponds to a choice of $z$ that makes the coefficient of the $M^{\prime} \wedge \bar{M}^{\prime}$ term in equation (4.40) real. When this choice has been made we have

$$
\mathrm{d} M^{\prime}=s a M^{\prime} \wedge \bar{M}^{\prime}+\mathrm{e}^{i t} M^{\prime} \wedge K^{\prime}+\bar{M}^{\prime} \wedge K^{\prime}
$$

where $a$ is a real function. Now, the remaining gauge freedom is

$$
M^{\prime}=s M \quad K^{\prime}=K+r\left(\mathrm{e}^{-i t / 2} \bar{M}+\mathrm{e}^{i t / 2} M\right),
$$

where $r$ is any real function. It is now convenient to change the variables from $(M, K)$ to $(N, K)$, where

$$
N=\mathrm{e}^{i t / 2} M .
$$

This choice is admissible since $t$ is an invariant. The forms $(N, K)$ are given up to transformations

$$
N^{\prime}=s N, \quad s= \pm 1, \quad K^{\prime}=K+r(N+\bar{N}),
$$

where $r$ is a real function. Equation (4.42) rewritten in terms of $\left(N^{\prime}, K^{\prime}\right)$ reads

$$
\mathrm{d} N^{\prime}=\frac{i}{2} \mathrm{~d} t \wedge N^{\prime}+s a \mathrm{e}^{i t / 2} N^{\prime} \wedge \bar{N}^{\prime}+\mathrm{e}^{i t}\left(N^{\prime}+\bar{N}^{\prime}\right) \wedge K^{\prime} .
$$

Thus we have a new equivalence problem for the forms $(N, K)$ which are given up to the transformations (4.45). The form $N^{\prime}$ satisfies (4.46). Writing the differential of $K$ in the most general form

$$
\mathrm{d} K=i b N \wedge \bar{N}+c N \wedge K+\bar{c} \bar{N} \wedge K
$$

and introducing the notation $\mathrm{d} t=t_{k} K+t_{+}(N+\bar{N})+t_{-} i(N-\bar{N})$ we easily find that

$$
\mathrm{d} K^{\prime}=s \omega^{0} \wedge\left(N^{\prime}+\bar{N}^{\prime}\right)+s\left[\frac{1}{2}(\bar{c}-c)+\frac{i}{2} r t_{k}\right] K^{\prime} \wedge\left(N^{\prime}-\bar{N}^{\prime}\right),
$$

where

$\omega^{0}=\mathrm{d} r-\left[2 r \cos t+\frac{1}{2}(c+\bar{c})\right] K+\frac{1}{2}\left[i\left(b-r t_{+}+2 r a \sin \frac{t}{2}\right)+r\left(\bar{c}-c+i r t_{k}\right)\right](N-\bar{N})+e(N+\bar{N})$,

and $e$ is an arbitrary real function on $\mathcal{N}$.

Now the following two cases may occur

ii.a1) $t_{k} \neq 0$ (i.e. $\mathrm{d} t \wedge N \wedge \bar{N} \neq 0$ )

ii.a2) $t_{k}=0$ (i.e. $\mathrm{d} t \wedge N \wedge \bar{N}=0$.) 
Case ii.a1) $(\mathrm{d} t \wedge N \wedge \bar{N} \neq 0)$

If $\mathrm{d} t \wedge N \wedge \bar{N} \neq 0$ then the choice

$$
r=\frac{c-\bar{c}}{i t_{k}}
$$

brings the system given by equations $(4.46),(4.48)$ to the form

$$
\begin{gathered}
\mathrm{d} N^{\prime}=\frac{i}{2} \mathrm{~d} t \wedge N^{\prime}+s a \mathrm{e}^{i t / 2} N^{\prime} \wedge \bar{N}^{\prime}+\mathrm{e}^{i t / 2}\left(N^{\prime}+\bar{N}^{\prime}\right) \wedge K^{\prime} \\
\mathrm{d} K^{\prime}=i b^{\prime} N^{\prime} \wedge \bar{N}^{\prime}+c^{\prime}\left(N^{\prime}+\bar{N}^{\prime}\right) \wedge K^{\prime},
\end{gathered}
$$

where $b^{\prime}$ and $c^{\prime}$ are appropriate functions on $\mathcal{N}$.

Now, we can return to the original variables $(M, K)$ instead of $(N, K)$. The result is summarized in the following proposition.

Proposition 6 A generic $N H S(\mathcal{N},[(M, K)])$ for which $I_{1}^{0}=\mathrm{e}^{i t}$, is equivalent to the one generated by forms $(M, K)$ which satisfy

$$
\begin{gathered}
\mathrm{d} M=s a M \wedge \bar{M}+\mathrm{e}^{i t} M \wedge K+\bar{M} \wedge K \\
\mathrm{~d} K=i s b M \wedge \bar{M}+s c\left[\mathrm{e}^{i t / 2} M+\mathrm{e}^{-i t / 2} \bar{M}\right] \wedge K .
\end{gathered}
$$

Here $\mathrm{d} t \wedge M \wedge \bar{M} \neq 0, a \geq 0, b$ and $c$ are real functions on $\mathcal{N}$. The full system of invariants in this case is given by $t, s a, s b, s c$ and their differentials. The local group of symmetries of such structures is at most of dimension 2.

(We note that the word "generic" in the above definition means "d $t \wedge M \wedge \bar{M} \neq 0$ ").

We close this section remarking that the forms $(M, K)$ are determined by conditions (4.54) uniquely modulo the sign of $M$. If not all the invariants $a, b, c$ are simultaneously zero, one can fix this freedom by requiring that $s a$ or $s b$ or $s \operatorname{Re} c$ or $s \operatorname{Im} c$ is greater than zero.

Case ii.a2) $(\mathrm{d} t \wedge N \wedge \bar{N}=0)$.

In this case equations (4.46), (4.48) take the form

$$
\begin{gathered}
\mathrm{d} N^{\prime}=\frac{i}{2} \mathrm{~d} t \wedge N^{\prime}+s a \mathrm{e}^{i t / 2} N^{\prime} \wedge \bar{N}^{\prime}+\mathrm{e}^{i t}\left(N^{\prime}+\bar{N}^{\prime}\right) \wedge K^{\prime}, \\
\mathrm{d} K^{\prime}=s \omega^{0} \wedge\left(N^{\prime}+\bar{N}^{\prime}\right)+s \frac{1}{2}[\bar{c}-c] K^{\prime} \wedge\left(N^{\prime}-\bar{N}^{\prime}\right) .
\end{gathered}
$$

The system (4.55) passes the Cartan test (with $n=3, r=1, r^{(1)}=1, s_{1}^{\prime}=1, s_{2}^{\prime}=0$, and $s_{3}^{\prime}=0$ ). Thus we conclude that it is gauge equivalent to the system

$$
\begin{gathered}
\mathrm{d} N^{\prime}=\frac{i}{2} \mathrm{~d} t \wedge N^{\prime}+s a \mathrm{e}^{i t / 2} N^{\prime} \wedge \bar{N}^{\prime}+\mathrm{e}^{i t}\left(N^{\prime}+\bar{N}^{\prime}\right) \wedge K^{\prime}, \\
\mathrm{d} K^{\prime}=s \frac{1}{2}[\bar{c}-c] K^{\prime} \wedge\left(N^{\prime}-\bar{N}^{\prime}\right) .
\end{gathered}
$$

Returning to the original variables $(M, K)$ we have the following proposition.

Proposition 7 A NHS $(\mathcal{N},[(M, K)])$ for which $I_{1}^{0}=\mathrm{e}^{i t}$, $\mathrm{d} I_{1}^{0} \wedge M \wedge \bar{M}=0, I_{1}^{0} \neq-1$ is equivalent to the one generated by the forms $(M, K)$ satisfying the following equations

$$
\begin{aligned}
\mathrm{d} M & =s a M \wedge \bar{M}+\mathrm{e}^{i t} M \wedge K+\bar{M} \wedge K \\
\mathrm{~d} K & =i s b\left[\mathrm{e}^{i t / 2} M \wedge K-\mathrm{e}^{-i t / 2} \bar{M} \wedge K\right],
\end{aligned}
$$


where $a$ and $b$ are real functions, $I_{1}^{0}=\mathrm{e}^{i t}$.

The invariants of the above structures are $a, b, t$ and their differentials. The sign ambiguity in the choice of $M$ can be removed by the requirements that $s a$ or $s b$ is greater than zero (provided that both $a$ and $b$ do not vanish).

The symmetry group of any of such structures is infinitely dimensional. The set of analytic selfequivalences for each structure depends on one real function of one variable.

As an example of null surfaces described by the above proposition all such structures for which $a, b$ and $t$ are constants are given in the following result:

Hypersurfaces for which $\left|I_{1}^{0}\right|=1, \mathrm{~d} I_{1}^{0} \wedge M \wedge \bar{M}=0, I_{1}^{0} \neq-1, \mathrm{~d} a=\mathrm{d} b=\mathrm{d} t=0$ have neccessarily $a=b=0$. Locally they can be described in a coordinate chart $(r, \xi, \bar{\xi})$ by the forms

$$
M=\mathrm{d} \xi+\left(\mathrm{e}^{i t} \xi+\bar{\xi}\right) \mathrm{d} r, \quad K=\mathrm{d} r, \quad t \neq \pi .
$$

Case ii.b) $(t=\pi)$.

If $t=\pi$ then equation 4.40 ) takes the form

$$
\mathrm{d} M^{\prime}=s[\bar{z}+z+\alpha-\bar{\beta}] M^{\prime} \wedge \bar{M}^{\prime}-M^{\prime} \wedge K^{\prime}+\bar{M}^{\prime} \wedge K^{\prime} .
$$

We fix $\operatorname{Re} z$ by demanding that the coefficient of the $M^{\prime} \wedge \bar{M}^{\prime}$ term is purely imaginary. Hence in this case we start with the forms $(M, K)$ such that

$$
\mathrm{d} M=i a M \wedge \bar{M}-M \wedge K+\bar{M} \wedge K,
$$

where $a$ is a nonnegative real function.

The remaining gauge freedom is

$$
M^{\prime}=s M, \quad s= \pm 1, \quad K^{\prime}=K+\operatorname{ir}(M-\bar{M}),
$$

where $r$ is arbitrary real function. Writing the differential of $K$ in the most general way

$$
\mathrm{d} K=i b M \wedge \bar{M}+c M \wedge K+\bar{c} \bar{M} \wedge K,
$$

where $b$ is real function, we get that following equations for the differentials of the lifted forms $\left(M^{\prime}, K^{\prime}\right)$.

$$
\begin{gathered}
\mathrm{d} M^{\prime}=i s a M^{\prime} \wedge \bar{M}^{\prime}-M^{\prime} \wedge K^{\prime}+\bar{M}^{\prime} \wedge K^{\prime}, \\
\mathrm{d} K^{\prime}=i s \omega^{0} \wedge\left(M^{\prime}-\bar{M}^{\prime}\right)+\frac{1}{2} s(c+\bar{c})\left(M^{\prime}+\bar{M}^{\prime}\right) \wedge K^{\prime} \\
\omega^{0}=\mathrm{d} r+\left[2 r+\frac{i}{2}(c-\bar{c})\right] K-\frac{1}{2}[b+i r(c++\bar{c})](M+\bar{M})+i u(M-\bar{M})
\end{gathered}
$$

where $u$ is a real function.

The above system passes Cartan's test with $n=3, r=1, r^{(1)}=1, s_{1}^{\prime}=1, s_{2}^{\prime}=s_{3}^{\prime}=0$. This leads to the following proposition.

Proposition 8 Any NHS $(\mathcal{N},[(M, K)])$ with $I_{1}^{0}=-1$ is gauge equivalent to one of the structures generated by the forms $(M, K)$ satisfying

$$
\begin{gathered}
\mathrm{d} M=i a M \wedge \bar{M}+K \wedge(M-\bar{M}), \\
\mathrm{d} K=b(M+\bar{M}) \wedge K,
\end{gathered}
$$

where $a$ and $b$ are real functions. If $a=0$ and $b=0$ the forms $(M, K)$ are defined by the above equations up to the sign of $M$, otherwise the forms are defined uniquely. 
The full set of invariants for such NHS's consists of the functions $a, b$ and their differentials. If $a \neq 0$ one can restrict to $a>0$. If $a=$ and $b \neq 0$ it is enough to consider $b>0$.

The symmetry group of such hypersurfaces is infinite dimensional. The set of all analytic self equivalences depends on one real function of one variable.

As an example of the hypersurfaces described by the above proposition we consider the case where $a$ and $b$ are constants. Then it follows that they both must be zero, and there exists a coordinate chart $(r, \xi, \bar{\xi})$ on such $(\mathcal{N},[(M, K)])$ such that

$$
M=\mathrm{d} \xi+(\xi-\bar{\xi}) \mathrm{d} r, \quad K=\mathrm{d} r .
$$

\subsection{Class 4: $\rho=0$ and $\sigma \neq 0$ on $\mathcal{N}$}

This case is very special because the conditions $\kappa=\rho=0$ on $\mathcal{N}$, together with the second structure Cartan equations (7.28) of [5] imply that

$$
\sigma \bar{\sigma}+S_{44} / 2=0 \quad \text { on } \quad \mathcal{N}
$$

Usually one is interested in the case where $\mathcal{N}$ is a hypersurface in a physically realistic space-time. Here we see that any hypersurface on which $\rho=0$ and $\sigma \neq 0$ can not exist in a space-time satisfying the dominant energy condition $S_{44} \geq 0$; hence, in particular, such NHS can not exist in any vaccum space-time.

If $\rho=0$ and $\sigma \neq 0$ then the coefficient of the $\bar{M}^{\prime} \wedge K^{\prime}$ term in equation (4.2) may be normalized to 1. Then we have

$$
\bar{\sigma} A^{-1} \mathrm{e}^{2 i \phi}=1
$$

which totally fixes $A$ and determines $\mathrm{e}^{i \phi}$ up to a sign. Hence in this case we can always start with forms $(M, K)$ which satisfy

$$
\mathrm{d} M=(\alpha-\bar{\beta}) M \wedge \bar{M}+(\varepsilon-\bar{\varepsilon}) M \wedge K+\bar{M} \wedge K
$$

The remaining gauge freedom is

$$
M^{\prime}=s M, \quad s= \pm 1, \quad K^{\prime}=K+z \bar{M}+\bar{z} M,
$$

where $z$ is a complex function.

We now proceed in the usual way. Calculating the differential of $M^{\prime}$ we get

$$
\mathrm{d} M^{\prime}=s[\bar{z}-z(\varepsilon-\bar{\varepsilon})+\alpha-\bar{\beta}] M^{\prime} \wedge \bar{M}^{\prime}+(\varepsilon-\bar{\varepsilon}) M^{\prime} \wedge K^{\prime}+\bar{M}^{\prime} \wedge K^{\prime} .
$$

Thus we see that the quantity $i J_{1}^{0}=\varepsilon-\bar{\varepsilon}$ is an invariant.

The following two cases are worth distinguishing.

a) $J_{1}^{0} \neq \pm 1$

b) $J_{1}^{0}= \pm 1$.

Case a) $J_{1}^{0} \neq \pm 1$.

In this case we can fix $z$ by

$$
z=\frac{-i \bar{J}_{1}^{0}(\alpha-\bar{\beta})+\bar{\alpha}-\beta}{\left|J_{1}^{0}\right|^{2}-1}
$$


Such a choice eliminates the $M^{\prime} \wedge \bar{M}^{\prime}$ term. The remaining freedom is only in the sign of $M$. The system reduces to

$$
\begin{array}{r}
\mathrm{d} M^{\prime}=i J_{1}^{0} M^{\prime} \wedge K^{\prime}+\bar{M}^{\prime} \wedge K^{\prime} \\
\mathrm{d} K^{\prime}=i J_{2}^{0} M^{\prime} \wedge \bar{M}^{\prime}+s J_{3}^{0} M^{\prime} \wedge K^{\prime}+s \bar{J}_{3}^{0} \bar{M}^{\prime} \wedge K^{\prime},
\end{array}
$$

where $J_{2}^{0}$ is a real function and $J_{3}^{0}$ is a complex function.

If $J_{3}^{0} \neq 0$ then we can fix the freedom totally by the requirement that its real or imaginary part is positive. Thus we have the following proposition.

Proposition 9 Any $N H S(\mathcal{N},[(M, K)])$ for which $\rho=0, \sigma \neq 0$ and $J_{1}^{0} \neq \pm 1$ is equivalent to one of the NHS's defined by the forms $(M, K)$ satisfying

$$
\begin{array}{r}
\mathrm{d} M=i J_{1}^{0} M \wedge K+\bar{M} \wedge K \\
\mathrm{~d} K=i J_{2}^{0} M \wedge \bar{M}+J_{3}^{0} M \wedge K+\bar{J}_{3}^{0} \bar{M} \wedge K,
\end{array}
$$

where $\operatorname{Re} J_{3}^{0} \geq 0$ (or $\operatorname{Im} J_{3}^{0} \geq 0$ if $\operatorname{Re} J_{3}^{0}=0$ ). The full set of invariants for such NHS's consists of $J_{1}^{0}, J_{2}^{0}, J_{3}^{0}$ and their differentials. The forms $(M, K)$ are defined by equation (4.73) uniquely if $J_{3}^{0} \neq 0$ and up to the sign of $M$ if $J_{3}^{0}=0$.

Every such NHS has symmetry group of dimension no greater than 3.

The classification of NHS's with $J_{1}^{0} \neq \pm 1$ and symmetry group of dimension 3 is given in Appendix A.

Case b) $J_{1}^{0}= \pm 1$.

Since $J_{1}^{0}$ is an invariant the null hypersurfaces belonging to the classes characterized by the opposite sign of $J_{1}^{0}$ are not equivalent. To find invariants in each of the two classes we proceed as follows. Equation (4.70) implies that

$$
\mathrm{d} M^{\prime}=s[\bar{z} \mp i z+\alpha-\bar{\beta}] M^{\prime} \wedge \bar{M}^{\prime} \pm i M^{\prime} \wedge K^{\prime}+\bar{M}^{\prime} \wedge K^{\prime} .
$$

Writing $z$ and $\alpha-\bar{\beta}$ in the form $z=\zeta+i \eta$ and $\alpha-\bar{\beta}=A+i B$, with $\zeta, \eta, A, B$ real, we see that the choice $z=i B+\zeta(1 \mp i)$ makes the coefficient of the $M^{\prime} \wedge \bar{M}^{\prime}$ term real. This shows that the present case can be generated by starting with forms $(M, K)$ satisfying

$$
\begin{gathered}
\mathrm{d} M=a M \wedge \bar{M} \pm i M \wedge K+\bar{M} \wedge K, \\
\mathrm{~d} K=i b M \wedge \bar{M}+c M \wedge K+\bar{c} \bar{M} \wedge K,
\end{gathered}
$$

where $a, b$ (real) and $c$ (in general complex) are functions. The remaining gauge freedom is

$$
M^{\prime}=s M, \quad s= \pm 1, \quad K^{\prime}=K+r[(1 \mp i) \bar{M}+(1 \pm i) M],
$$

where $r$ is a real function.

The differentials of $\left(M^{\prime}, K^{\prime}\right)$ read

$$
\begin{gathered}
\mathrm{d} M^{\prime}=s a M^{\prime} \wedge \bar{M}^{\prime} \pm i M^{\prime} \wedge K^{\prime}+\bar{M}^{\prime} \wedge K^{\prime}, \\
\mathrm{d} K^{\prime}=s \omega^{0} \wedge\left[(1 \pm i) M^{\prime}+(1 \mp i) \bar{M}^{\prime}\right]+ \\
\frac{1}{4} s[c(1 \mp i)-\bar{c}(1 \pm i)]\left[(1 \pm i) M^{\prime}-(1 \mp i) \bar{M}^{\prime}\right] \wedge K^{\prime},
\end{gathered}
$$

where

$$
\begin{gathered}
4 \omega^{0}=4 \mathrm{~d} r+[i b \pm 2 i a r-c r(1 \mp i)+\bar{c} r(1 \pm i)][(1 \pm i) M-(1 \mp i) \bar{M}] \\
-[c(1 \mp i)+\bar{c}(1 \pm i)] K+u[(1 \pm i) M+(1 \mp i) \bar{M}]
\end{gathered}
$$

and $u$ is arbitrary real constant. We easily verify that the system (4.77) passes the Cartan test $\left(n=3, r=1, r^{(1)}=1, s_{1}^{\prime}=1, s_{2}^{\prime}=s_{3}^{\prime}=0\right)$, hence we have the following proposition. 
Proposition 10 Every $N H S(\mathcal{N},[(M, K)])$ for which $J_{1}^{0}= \pm 1$ is gauge equivalent to one of the null hypersurfaces generated by forms $(M, K)$ with differentials

$$
\begin{gathered}
\mathrm{d} M=a M \wedge \bar{M} \pm i M \wedge K+\bar{M} \wedge K, \\
\mathrm{~d} K=b[(1 \pm i) M-(1 \mp i) \bar{M}] \wedge K,
\end{gathered}
$$

where $a \geq 0, b$ are real functions ( $b \geq 0$ if $a=0$ ).

The full set of invariants for such NHS's is given by $a, b$ and their differentials.

The forms $(M, K)$ are defined uniquely if at least one of $a$ and $b$ does not vanish. Otherwise the forms are defined up to a sign of $M$.

The symmetry group for such NHS's is infinite dimensional. The set of all analytic self-equivalences for each NHS depends on one real function of one variable.

An example of a surface belonging to the class described by the above proposition is a null hypersurface structure defined by forms

$$
M=\mathrm{d} \xi+(\bar{\xi} \pm i \xi) \mathrm{d} r, \quad K=\mathrm{d} r,
$$

on a surface $\mathcal{N}$ coordinatized by $(r, \xi, \bar{\xi})$.

All the major results of this section and those of Appendix A are summarized in a diagram in Appendix B.

\section{Conclusions}

We have investigated the intrinsic geometry of null hypersurfaces by formulating the concept of a null hypersurface structure. All such structures have been classified into equivalence classes by using Cartan's method which is adapted to the use of differential forms. The resulting classification can be used in the study of gravitational problems involving null hypersurfaces. The techniques that have been used could also be applied to null hypersurfaces in higher dimensional space-times. Here we have concentrated on classifying a structure which is equivalent to Penrose's I - geometry. It would be interesting and informative to classify II - and II - geometries and to compare the solutions of the corresponding equivalence problems. 


\section{A NHS's with 3-dimensional symmetry groups.}

Here we explicitly compute the details of one of the classes specified in Proposition 9 of Section 4 . We present all null NHS's for which $\rho=0, \sigma \neq 0$ and the symmetry group is strictly 3-dimensional. They have the property that all the invariants $J_{1}^{0}, J_{2}^{0}, J_{3}^{0}$ are constants.

By taking the exterior derivative of equations (4.73) we obtain $J_{3}^{0}=0$. Thus such NHS'sare defined by the system (cf. equation (4.73))

$$
\begin{array}{r}
\mathrm{d} M=i J_{1}^{0} M \wedge K+\bar{M} \wedge K \\
\mathrm{~d} K=i J_{2}^{0} M \wedge \bar{M},
\end{array}
$$

with real constants $J_{1}^{0}$ and $J_{2}^{0}$. The following cases may occur.

1) $J_{2}^{0} \neq 0$

1a) $\left(-J_{2}^{0}, J_{1}^{0}-1, J_{1}^{0}+1\right)$ have all the same sign $s$.

In this case the symmetry group is of Bianchi type $I X$, and the forms $(M, K)$ may be choosen in such a way that

$$
M=\frac{s(1+i)}{2 \sqrt{-J_{2}^{0}\left(J_{1}^{0}-1\right)}} \theta^{1}+\frac{1-i}{2 \sqrt{-J_{2}^{0}\left(J_{1}^{0}+1\right)}} \theta^{2}, \quad K=\frac{1}{\sqrt{\left(J_{1}^{0}\right)^{2}-1}} \theta^{3},
$$

where the forms $\left(\theta^{1}, \theta^{2}, \theta^{3}\right)$ in a suitable coordinate system $(u, x, y)$ are given by

$$
\begin{gathered}
\theta^{1}=\cos y \cos u \mathrm{~d} x-\sin u \mathrm{~d} y \\
\theta^{2}=\cos y \sin u \mathrm{~d} x+\cos u \mathrm{~d} y \\
\theta^{3}=-\sin y \mathrm{~d} x+\mathrm{d} u .
\end{gathered}
$$

We have a 2-parameter family of nonequivalent NHS's here.

1b) not all $\left(-J_{2}^{0}, J_{1}^{0}-1, J_{1}^{0}+1\right)$ have the same sign.

In this case the symmetry group is of Bianchi type $V I I I$, so it is convenient to introduce a coordinate chart $(x, y, u)$ on $\mathcal{N}$ and forms

$$
\begin{gathered}
\Theta^{1}=\cosh y \cos u \mathrm{~d} x-\sin u \mathrm{~d} y \\
\Theta^{2}=\cosh y \sin u \mathrm{~d} x+\cos u \mathrm{~d} y \\
\Theta^{3}=\sinh y \mathrm{~d} x+\mathrm{d} u .
\end{gathered}
$$

The following three cases may occur.

1bi) $J_{1}^{0}+1=s\left|J_{1}^{0}+1\right|, J_{1}^{0}-1=s\left|J_{1}^{0}-1\right|, J_{2}^{0}=s\left|J_{2}^{0}\right|, s= \pm 1$.

In this case the null hypersurface structure is generated by $(M, K)$ such that

$$
M=\frac{s(1+i)}{2 \sqrt{J_{2}^{0}\left(J_{1}^{0}-1\right)}} \Theta^{1}+\frac{1-i}{2 \sqrt{J_{2}^{0}\left(J_{1}^{0}+1\right)}} \Theta^{2}, \quad K=\frac{1}{\sqrt{\left(J_{1}^{0}\right)^{2}-1}} \Theta^{3} .
$$

1bii) $J_{1}^{0}+1=s\left|J_{1}^{0}+1\right|, J_{1}^{0}-1=-s\left|J_{1}^{0}-1\right|, J_{2}^{0}=s\left|J_{2}^{0}\right|, s= \pm 1$.

In this case the null hypersurface structure is generated by $(M, K)$ such that

$$
M=\frac{s(1+i)}{2 \sqrt{-J_{2}^{0}\left(J_{1}^{0}-1\right)}} \Theta^{3}+\frac{1-i}{2 \sqrt{J_{2}^{0}\left(J_{1}^{0}+1\right)}} \Theta^{2}, \quad K=\frac{1}{\sqrt{1-\left(J_{1}^{0}\right)^{2}}} \Theta^{1} .
$$


1biii) $J_{1}^{0}+1=s\left|J_{1}^{0}+1\right|, J_{1}^{0}-1=-s\left|J_{1}^{0}-1\right|, J_{2}^{0}=-s\left|J_{2}^{0}\right|, s= \pm 1$.

In this case the null hypersurface structure is generated by $(M, K)$ such that

$$
M=\frac{1+i}{2 \sqrt{J_{2}^{0}\left(J_{1}^{0}-1\right)}} \Theta^{2}+\frac{s(1-i)}{2 \sqrt{-J_{2}^{0}\left(J_{1}^{0}+1\right)}} \Theta^{3}, \quad K=\frac{1}{\sqrt{1-\left(J_{1}^{0}\right)^{2}}} \Theta^{1} .
$$

In all cases $1 \mathrm{~b})$ we have 2-parameter families of inequivalent NHS's.

2) $J_{2}^{0}=0$

2a) $\left|J_{1}^{0}\right|<1$.

In this case the symmetry group is of Bianchi type $V I_{0}$, and the forms $(M, K)$ may be chosen in such a way that

$$
M=\frac{1+i}{\sqrt{2}} \sqrt{\frac{1+J_{1}^{0}}{1-J_{1}^{0}}} \tau^{1}+\frac{1-i}{\sqrt{2}} \tau^{2}, \quad K=\frac{1}{\sqrt{1-\left(J_{1}^{0}\right)^{2}}} \tau^{3},
$$

where, in appropriate coordinates, the forms $\left(\tau^{1}, \tau^{2}, \tau^{3}\right)$ are

$$
\begin{gathered}
\tau^{1}=\cosh x \mathrm{~d} y-\sinh x \mathrm{~d} u \\
\tau^{2}=-\sinh x \mathrm{~d} y+\cosh x \mathrm{~d} u \\
\tau^{3}=\mathrm{d} x .
\end{gathered}
$$

It follows that we have a one-parameter family of nonequivalent NHS's here.

2b) $\left|J_{1}^{0}\right|>1$

In this case the symmetry group is of Bianchi type $V I I_{0}$, and the forms $(M, K)$ may be chosen in such a way that

$$
M=\frac{1+i}{\sqrt{2}} \sqrt{\frac{J_{1}^{0}+1}{J_{1}^{0}-1}} \sigma^{1}+\frac{1-i}{\sqrt{2}} \sigma^{2}, \quad K=\frac{1}{\sqrt{\left(J_{1}^{0}\right)^{2}-1}} \sigma^{3},
$$

where, in appropriate coordinates, the forms $\left(\sigma^{1}, \sigma^{2}, \sigma^{3}\right)$ are

$$
\begin{gathered}
\sigma^{1}=\cos x \mathrm{~d} y-\sin x \mathrm{~d} u \\
\sigma^{2}=\sin x \mathrm{~d} y+\cos x \mathrm{~d} u \\
\sigma^{3}=\mathrm{d} x .
\end{gathered}
$$

It follows that here we have a one-parameter family of inequivalent null hypersurfaces.

We close this appendix by remarking that the NHS's described here and in Proposition 5 exhaust all the cases of NHS's with strictly 3-dimensional group of symmetries. All the other NHS's have either infinite dimensional symmetry groups or have symmetry groups of dimension no greater than 2 . 


\section{B The classification of Section 4 and Appendix A}

Below we present a tree of the classification obtained in the preceeding sections.

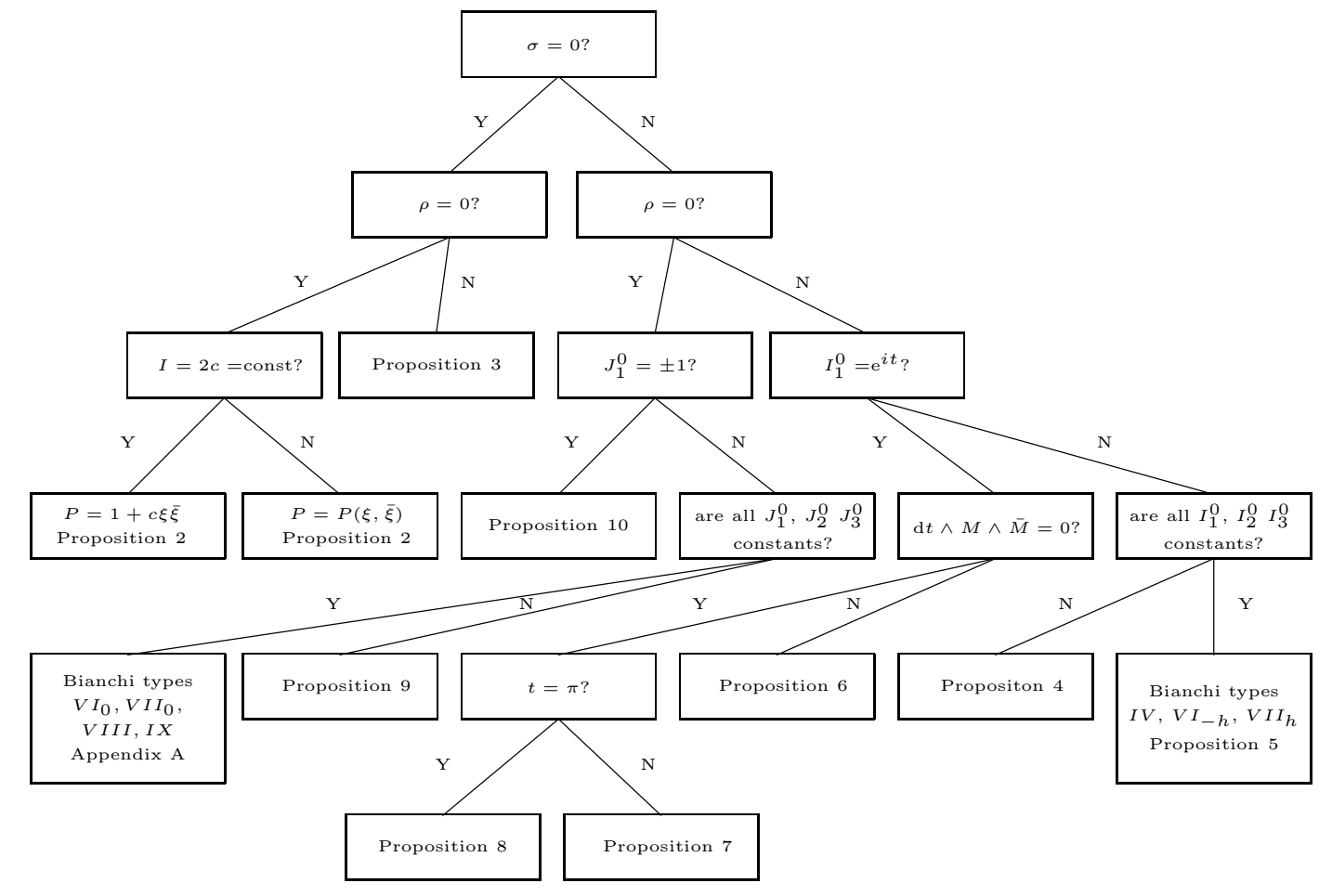

\section{References}

[1] Ashtekar A, Beetle Ch, Dreyer O, Fairhurst S, Krishnan B, Lewandowski L, Wiśniewski J 2000, "Isolated horizons and their applications" preprint gr-qc/0006006

[2] Brans C H 1965 "Invariant approach to the geometry of spaces in general relativity", Journ. Math. Phys. 6, $94-102$

[3] Burdet G, Perrin M 1997 "Radiation structures on isotropic hypersurfaces", preprint: dgga/9704014

[4] Karlhede A 1980 "A review of the geometrical equivalence of metrics in general relativity" Gen. Rel. Grav. 12, 693 -707

[5] Kramer D, Stephani H, MacCallum M, Herlt E 1980 Exact solutions of Einstein's field equations, Deutscher, Berlin

[6] Olver P J 1996 Equivalence, invariants and symmetry, Cambridge Univ. Press, Cambridge

[7] Penrose R 1972, "The geometry of impulsive gravitational waves" in General Relativity ed. L. O’Raifeartaigh, Clarendon, Oxford, pp. 101-115 